\title{
Folk astronomy in Omani agriculture
}

\author{
Harriet Nash and Dionisius A. Agius \\ Institute for Arab and Islamic Studies, University of Exeter, \\ Stocker Road, Exeter, EX4 4ND, United Kingdom \\ email: nashalbu@uku.co.uk D.A.Agius@exeter.ac.uk
}

\begin{abstract}
The Sultanate of Oman is possibly unique in using stars to time the allocation of water in the traditional irrigation systems called aflāj (s. falaj), which are still an important part of Omani culture and sense of national identity. This is a rapidly disappearing oral tradition that has hardly been known about outside Oman prior to this study. The need for timing water shares is explained and the methods of stargazing described. The identities of the stars, their Omani names and the relationship with modern star names are discussed.
\end{abstract}

Keywords. Oman, stargazing, timing water, agriculture, star names

\section{Introduction}

The use of stars in timing irrigation water appears to be carried out only in Oman, where traditional irrigation systems called aflāj (s. falaj) are still an important part of community life. Rainfall in the northern mountains is high enough to recharge groundwater, which is tapped by aflāj and used for irrigation away from the mountains, in an otherwise arid area (Fig. 1). Date palms are the most important crop. The falaj system comprises a water source (usually a spring or groundwater), channels for transporting the water and for distributing it within the irrigated area, all by gravity flow. Archaeological excavations have established that these systems may date from 3000 BCE (Orchard \& Orchard 2007).

Because the water flows under gravity, it does not stop at night, and being a precious resource is used for the full 24 hours. The water-time shares owned by many people are less than 30 minutes, so there is a usually a need to time the distribution. Before the introduction of wristwatches in the late 1960s, most communities used a sundial by day and stars by night for timing the shares. Currently there are about 3,000 operational falaj systems, many still using the sundial, but only about eight of them using stars. There is practically nothing written on these traditional timing systems: the knowledge is handed orally down from generation to generation. This paper is, therefore, based almost entirely on Nash's field work for her PhD thesis (Nash 2009) in the period 2005-2008†, which was supervised by Professor D.A. Agius. The research could not have taken place without the unstinting support and assistance of the falaj stargazers and the Ministry of Water Resources in Oman.

\section{Traditional timing of water shares}

The basic division of time is into day and night, each of which is divided into 24 athars, one athar in theory averaging $\frac{1}{2}$ hour over the year, although in some villages the sahm ( $1 \frac{1}{2}$ athars) is preferred. The sundials are mostly of the form of that in Fig. 2: a pole with

$\dagger$ Partly funded by grants from the Arts and Humanities Research Council and the Seven Pillars of Wisdom Trust. 


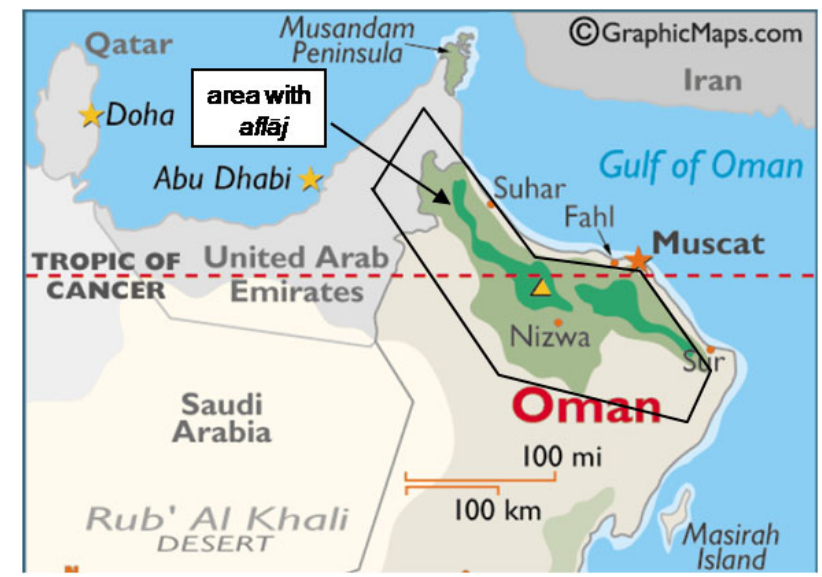

Figure 1. Location of Oman (courtesy of GraphicMaps.com).

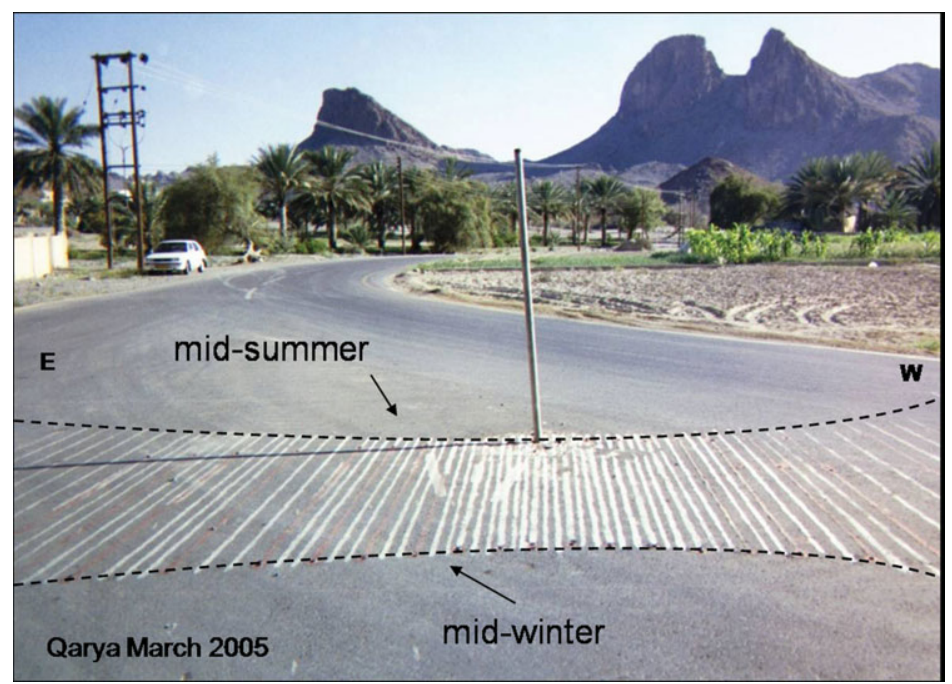

Figure 2. Sundial at Qarya, Northern Oman, showing the path traced by the pole's shadow.

lines or other markers on the ground. When the shadow of the tip of the pole reaches the marker for the start of someone's water share, they go to the falaj channel(s) and move the sluices to change the direction of flow to their fields.

The simplest method of stargazing is to watch the stars either setting below or rising above the natural horizon, the method used in the village of Qarya. Again, each person is responsible their own timing and when it is time, they direct the water to their fields as for the sundial. People with more experience are available to resolve any disputes over both day and night water allocation. When visibility is poor due, for example, to clouds and dust, the shadow of the sundial pole can still usually be seen, but the stars normally used may not be visible. In such conditions the time divisions may be estimated from experience, the use of alternative stars in a clearer part of the sky, or, more frequently, by the use of wristwatches. 


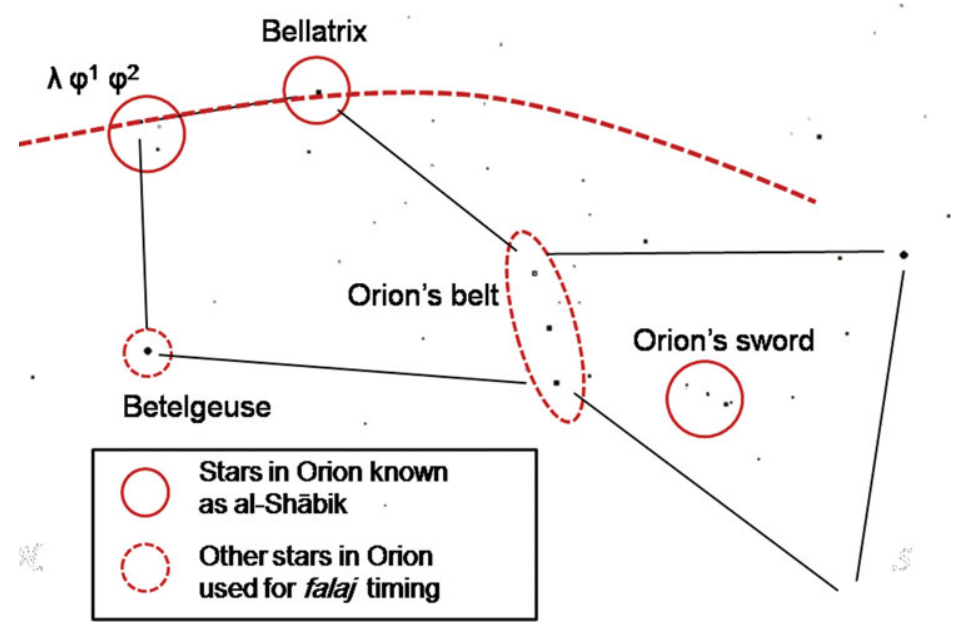

Figure 3. Stars in Orion called al-Shābik in Oman.

\section{Star names}

A major achievement of the research is to identify, for the first time, most of the stars used in different villages in Oman for timing irrigation water. Several falaj star namest differ from those for the same star in Arabic literature (e.g. the 9th-10th century savant al-Șüfi, Kunitzsch (1961)). This is not surprising given that star names are rarely unique, appearing in more than one constellation. Omani farmers may use a name appearing in the literature, but often for a different star: their knowledge is from an oral tradition, and they have no need of names from books The internationally used star name Algenib, for example, is for $\gamma$ Pegasus, magnitude 2.81; the name Algenib being taken from the Arabic al-Janb (meaning the side or flank) for this star. However in Oman, al-Janb is a few stars in the head of Hydra, the brightest of which is $\epsilon$ Hydrae at a magnitude of $3.37 \ddagger$. That said, several star names used in Oman are found in the literature dating to pre-Islamic times, including Kuwī (fiery brand), the old Arabic name for Vega ( $\alpha$ Lyrae) (Kunitzsch 1983), the modern name being al-Nasr al-Wāqic (the perched eagle).

In Oman and the rest of the Arab world the same name is often given to two separate stars rising at about the same time. They may be differentiated as North or South, high or low or whatever, but since the stargazers in Oman usually only use one for falaj timing, they do not always distinguish them, sometimes leading to confusion over the star's identity. Al-Sha ${ }^{c}$ ra comprises Procyon and Sirius in Oman and elsewhere, but Procyon is generally preferred for falaj purposes. Al- ${ }^{c}$ Aqrab (the scorpion) is generally the constellation of Scorpius in the literature; in Oman, the name al- ${ }^{c}$ Aqrab is applied in places to the first two or three stars of Scorpius $(\beta, \delta, \pi)$ to rise (Antares ( $\alpha$ Scorpii) is not included in the constellation). However, in at least one village (Qarya), there is a northern ${ }^{c}$ Aqrab-Eltanin ( $\gamma$ Draconis). Al-Debrān (the follower of Thurrayya, the Pleiades) is usually Aldebaran (sometimes with additional stars in the head of Taurus), as in the literature, but Capella is treated as its pair and is also known by this name in some villages.

An example of using a falaj star name for different stars in different villages is shown in Fig. 3. Al-Shābik (the fishing net), is seen in one place literally as a net bounded at the

$\dagger$ A falaj "star" can be one or more stars considered as a single item. Some of these may be far apart, and the term "asterism" is, therefore, often not appropriate.

$\ddagger \zeta$ Hydræ, magnitude 3.09, may also be/have been used in places. 


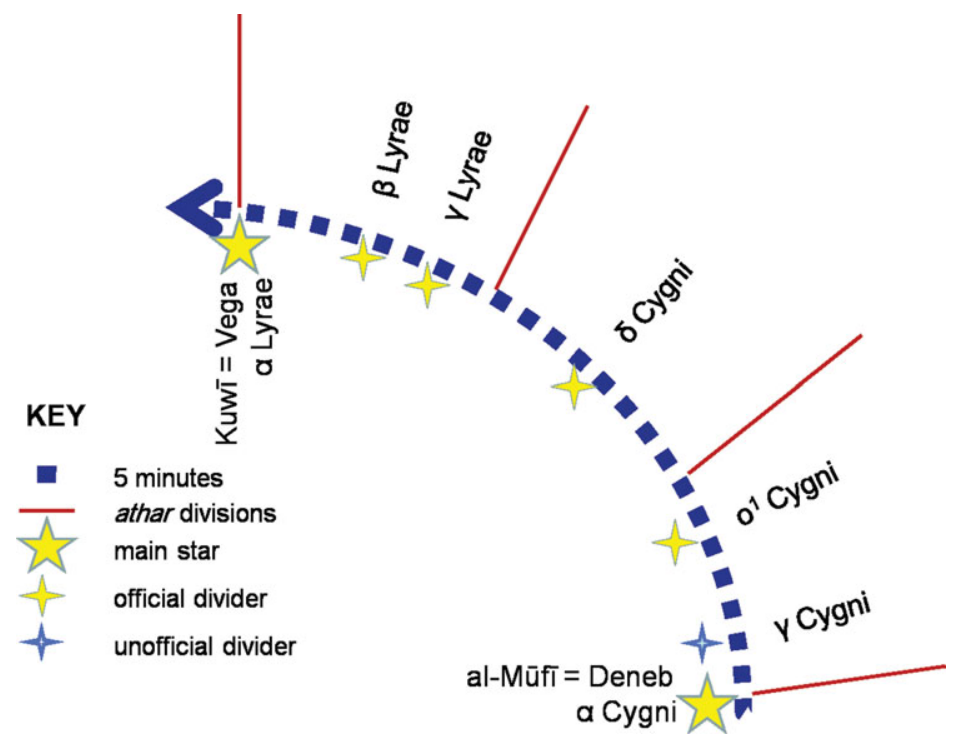

Figure 4. Athar divisions and divider stars in Qarya.

top by the dashed curve, with Bellatrix ( $\gamma$ Orionis), close to the apex, being the afalaj star. Elsewhere it is $\lambda, \phi^{1}, \phi^{2}$ Orionis, the same stars as a lunar station of the Islamic calendar called al-Haq ${ }^{c}$ a, and in Barzaman, the furthest south of the villages still using stars, it is Orion's sword. In one isolated place in the mountains, Coma Berenices is called al-Shābik, but is not used for the falaj divisions. This is unusual in that most farmers do not name stars that they do not use. These examples show that it is not possible to identify the stars used for agriculture by name alone: they must be observed to be sure.

\section{Methods of stargazing}

The simplest method of stargazing is to watch the stars either setting below or rising above the natural horizon, as in the village of Qarya where twenty one main stars are used in the course of a year, with 2 to 3 athars, that is approximately 1 to 1.5 hrs between each star. Many divider stars are used to obtain shorter divisions, but the dividers are not given names even though they may be bright stars, such as Betelgeuse ( $\alpha$ Orionis) and Antares ( $\alpha$ Scorpii). Fig. 4 illustrates that the divisions of time can be complex: there are 3 athars between the rise of Vega ( $\alpha$ Lyrae) and Deneb ( $\alpha$ Cygni): and for equal divisions of the night the maximum length of each athar should be about 33 minutes, but the first two are 40 minutes third 35 minutes long, and the divider stars bear little relation to the athar divisions.

Another method is to watch stars rising above a man-made horizon, such as a wall, close to the observer: time can be divided by moving closer to the wall, watching the same star rise at a later time. In this case divider stars are not needed. In built up areas, this method has developed further: indicator stars, which give the time of rise of the main falaj stars above the natural horizon are watched rising above or setting below different markers, and again the observer moves seeing the same indicator star rising or setting at a later time, with observation points for the athars, two to each falaj star $\dagger$ marked on walls, as illustrated in Fig. 5. Here, Sirius ( $\alpha$ Canis Majoris) is watched rising

$\dagger$ in the villages where this method is used there are 24 main falaj stars. 


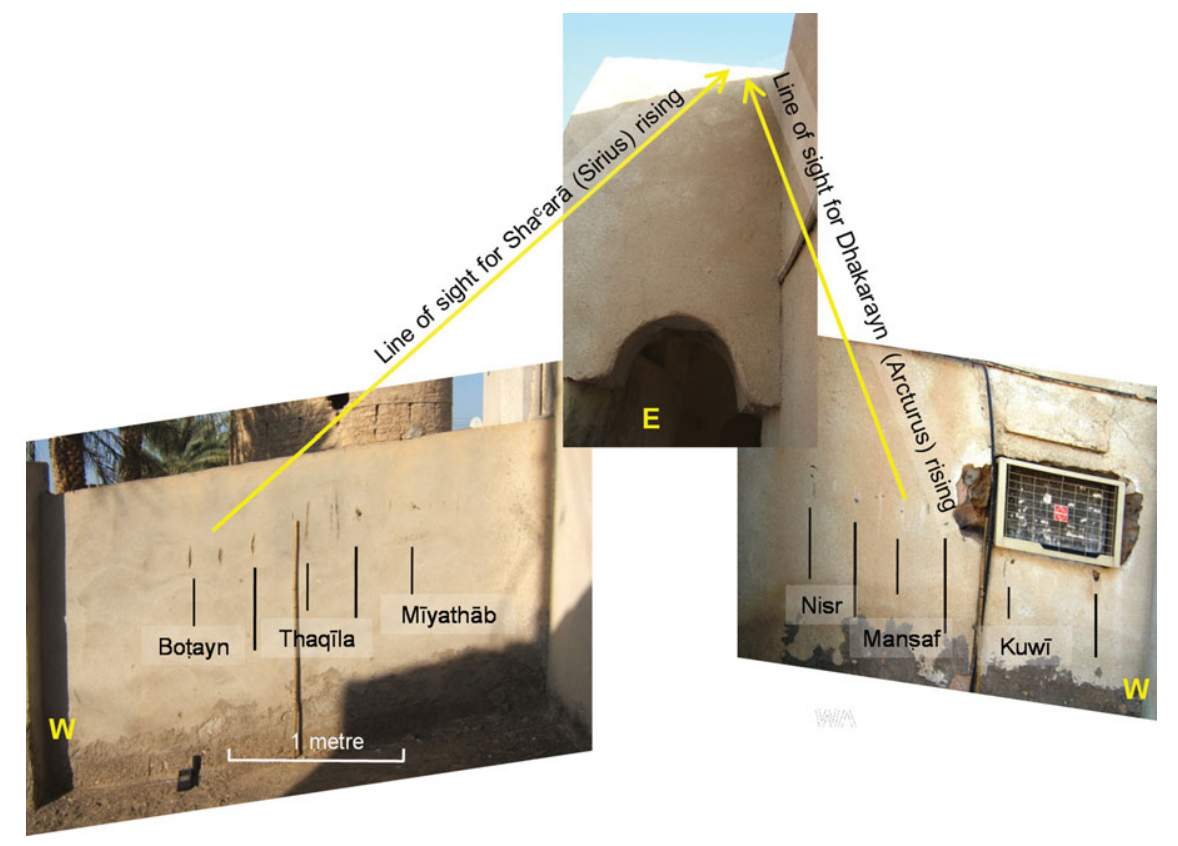

Figure 5. Walls used for stargazing in Al Fath.

for three stars from the north wall of a mosque compound, while Arcturus ( $\alpha$ Boötis) is watched for three stars from the south wall. For this method, there are 40 or so places to watch from, but it is only necessary to know six or seven stars: usually the brightest and most easily recognized, which makes the method relatively easy for farm workers to use unaided.

Some of the main falaj stars from different villages are shown in Fig. 6. They are mostly grouped about $27^{\circ} \mathrm{N}$, the latitude of the part of Oman where they are used. They bear little relationship to the lunar stations of the Islamic calendar also shown. The lunar stations are, generally speaking, stars where the moon is seen on consecutive nights. They roughly follow the ecliptic, and are more equally spaced in time than the falaj stars. Although stargazing for falaj water was probably carried out long before the lunar stations were introduced to Arabia from India, one might have expected the farmers to select stars at more equal spaces of time. On the other hand, since the stars used at a particular time of night change every week or so, the time shares even out over the year.

\section{The dying art of stargazing}

Despite many falaj systems being abandoned, mainly because of reduced flow due to pumped wells lowering groundwater levels, there are c. 3,000 operating systems and the falaj still forms an important part of the sense of Omani identity. This is one of the reasons that UNESCO recently registered some aflāj as a World Heritage Site (Al Sulaimani et al. 2007). However, only in eight falaj communities are stars still used. In addition to the introduction of wristwatches in the late 1960s, light pollution has been a major factor in the decline of stargazing. The younger generation has access to education and work outside the village; few are learning the ancient art of stargazing. As those with knowledge age and their eyesight fades, wristwatches become the preferred means 


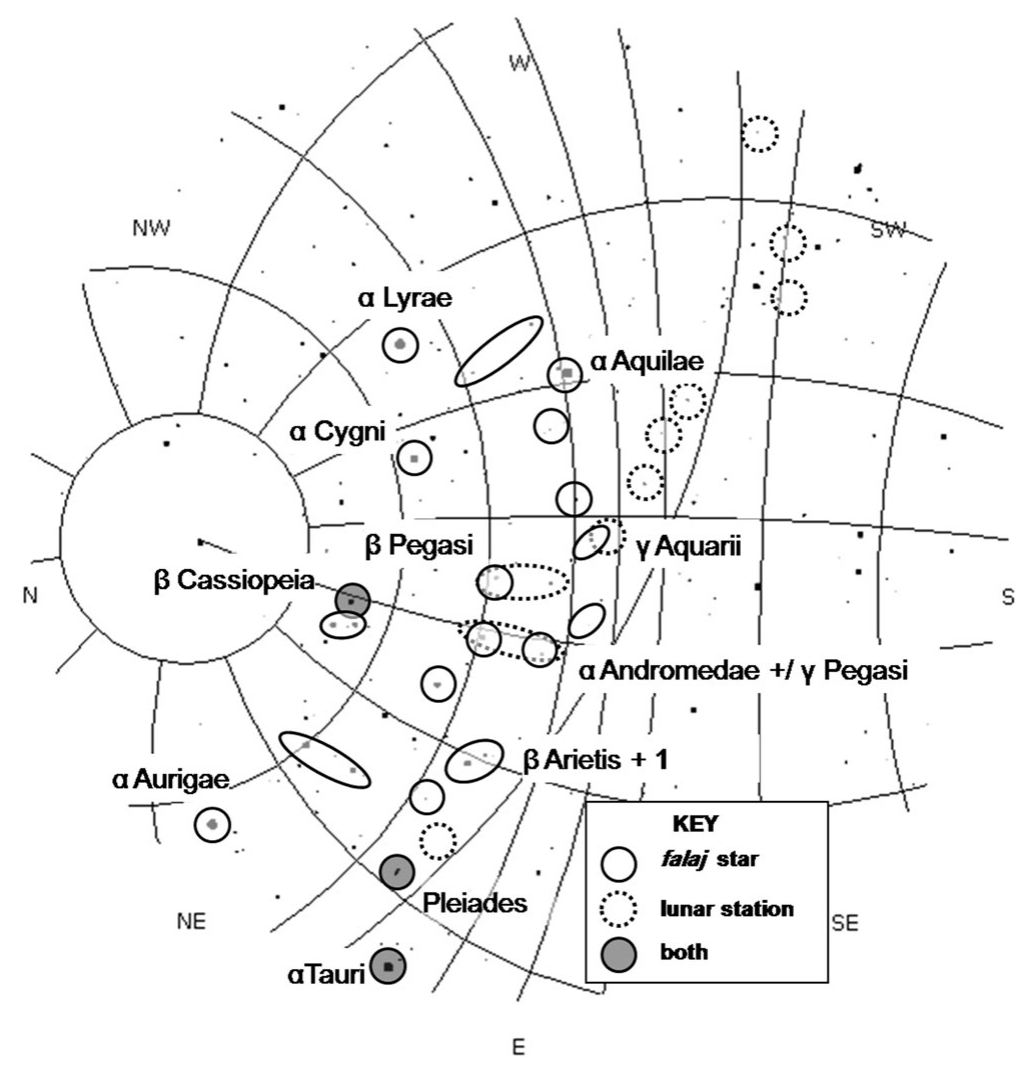

Figure 6. Locations of some falaj stars.

of timing water shares; it seems unlikely that this practice will continue for more than about 20 years.

The research that this paper is based on therefore represents a significant contribution to the understanding of a now rare use of stars, and of Oman's cultural heritage.

\section{References}

Al Sulaimani, Z., Helmi, T., \& Nash, H. 2007, The social importance and continuity of falaj use in northern Oman, International History Seminar on Irrigation and Drainage, 2-5 May 2007 Tehran http://hdl.handle.net/10036/15174

Kunitzsch, P. 1961, Untersuchungen zur Sternomenklatur der Araber (Wiesbaden: Harrasowitz Verlag)

Kunitzsch, P. 1983, Über eine anwā'-Tradition mit bisher unbekannten Sternnamen (Munich: Bavarian Academy of Science and C.H. Beck Verlag)

Nash, H. 2009, Water management: the use of stars in Oman, PhD thesis (Exeter: Institute of Arab and Islamic Studies, University of Exeter)

Orchard, J. \& Orchard, J. (eds) 2007, in Proceedings of the International Symposium, Archaeology of the Arabian Peninsula Through the Ages 7th-9th May 2006. Monograph No. 134. (Muscat: Ministry of Heritage and Culture) 